meant by "more effective." This unqualified assertion is likely to confuse the many people who already use or recommend All-Bran as an "effective" means of avoiding constipation whatever its soluble:insoluble fibre ratio or influence on plasma lipids, mineral absorption, faecal bulking, bowel transit time, etc. Furthermore, it is difficult to see why "wholemeal bread and crispbreads" are the only items to be recommended for a weight reducing diet from a list which includes acceptable alternatives such as granary bread, Hovis, HiBran, and Vitbe (presumably Vitbe Wheatgerm). Appreciation of patient preference appears to be totally forsaken in these inflexible recommendations for breakfast cereals and bread, which thus ignore the possibility of including suitable second best choices in an attempt to encourage dietary compliance.

An important opportunity to stimulate the growing interest in nutrition among medical practitioners has been missed. Nutrition educators who hope to be heeded must avoid the traditionally condescending attitude which is scattered throughout this series of articles.

Rowett Research Institute,

Aberdeen AB2 9SB

ANNE BRUCE

Department of Medicine,

K C MCHARDY

Aberdeen University,

Aberdeen

\section{Recurring meningitis: beware the normal looking ear}

SIR,--It seems that Mr Maurice Hawthorne (6 December, p 1504) has shared the same difficulties in detecting the source of infection in recurring meningitis. Scintigraphy using injected indium-111 diethylenetriamine pentacetic acid (DTPA) is a valuable diagnostic tool, but we have found computed tomography with intrathecal contrast to be equally sensitive.

In our neuroradiology department the commonest application of scintigraphy is in detecting suspected communicating hydrocephalusfor example, after meningitis or subarachnoid haemorrhage-and in investigating the patency of cerebrospinal fluid shunts. We agree that scintigraphy is very useful in investigating cerebrospinal rhinorrhoea after a fracture of the base of the skull or destructive lesions affecting the floor of the anterior cranial fossa. However, when faced with recurring meningitis in which an otological cause is suspected together with an intact and normal tympanic membrane then computed tomography with intrathecal contrast is the investigation of choice and we have detected several small leaks using this method.

Furthermore, many cerebrospinal fluid leaks often stop spontaneously, albeit temporarily, and this only adds greater difficulty in diagnosis.

L DURHAM

I J MACKENZIE

Department of Otorhinolaryngology, Walton Hospital,

Liverpool 9

\section{Randomised trial of treatment of hypertension in elderly patients in primary care}

SIR,-May we thank Dr Mark McCarthy (13 December, p 1570) for pointing out that the difference between the proportion of smokers in our treatment and control group was statistically significant. These smoking habits were recorded before randomisation, and therefore the difference was not due to a change of behaviour consequent on the treatment group to which the patients were assigned.

Could this asymmetry have contributed to the excess of deaths from cancer in the treatment group? Five of the 17 patients who died from cancer in the treatment group smoked as against two of the 10 in the control group. Since the study terminated in August 1985 there have been four more deaths from cancer, all cancers of the lung. Three of these were in the control group and one in the treatment group. This gives total numbers of deaths from cancer of 18 in the treatment group and 13 in the control group. It suggests more the tricks of the goddess of chance than any behaviour modification demanding a "paradigm shift."

With regard to observer bias in the study this is not likely to have much affected fatal strokes, where a significant difference was found. The records of all the patients were examined in the practice centres every six months by outside observers and all events were adjudicated by an independent committee that was blinded to the treatment status. Random zero sphygmomanometers were used to reduce bias in the measurement of blood pressure.

JOHN R COOPE THOMAS S WARRENDER

Bollington,

Near Macclesfield SK10 5JL

\section{Optimising antiemesis in cancer} chemotherapy

SIR,-The finding by Ms Pamela S Warrington and colleagues that metoclopramide needs to be given in a continuous infusion to provide effective antiemesis (22 November, p 1334) is in keeping with our studies showing its brevity of action. ${ }^{1}$ We found that a single $10 \mathrm{mg}$ dose did not last long enough to control postoperative sickness after preoperative morphine.

We have reported the undoubted efficacy of a single acupuncture treatment at the antiemetic point P6 (Neiguan) in reducing the emetic sequelae of opioids, ${ }^{34}$ and this work has been extended at the Northern Ireland radiotherapy centre to control nausea and vomiting after chemotherapy. A survey of 71 patients attending the outpatient clinic showed that 54 had distressing emetic sequelae on their first treatment, of whom 52 had the same symptoms on subsequent occasions. Our studies were carried out in patients who had experienced severe sickness on a previous occasion.

We treated 14 inpatients receiving cisplatin with five minutes' electrical acupuncture $(10 \mathrm{~Hz})$ at P6 point. The first was given immediately before injection of the drug, with others on the following day at the request of the patients. All had had prolonged and severe sickness after previous treatments. Eleven patients had complete absence of emetic symptoms for at least eight hours while three had some improvement. Acupuncture was performed up to six times in many patients, with beneficial effects on each occasion. This covered the expected duration of the emetic effect of cisplatin in most patients. Unknown to them five of these patients had one "dummy" acupuncture during the course of treatment and this produced no benefit.

In addition 29 assorted outpatients, who had been sick after previous cancer chemotherapy, had one to five acupuncture treatments. Twenty one of these had complete alleviation of emetic symptoms four had considerable improvement compared with their previous treatment, and four had some improvement.

In our experience the beneficial effect of a single $10 \mathrm{~Hz}$ electroacupuncture lasts for eight to 10 hours. We have found no side effects for this treatment but it is time consuming. Further work is needed to evaluate non-invasive methods of acupuncture and, we hope, evolve a method which patients could use at home.

J W DUNDEE R G GHALY K T J FITZPATRICK

Department of Anaesthetics, Queen's University of Belfast, Belfast BT9 7BL

G LYNCH

P ABRAM

Northern Ireland Radiotherapy Centre, Belfast

1 Assaf RAE, Clarke RSJ, Dundee JW, Samuel IO. Studies of drugs given before anaesthesia XXIV: Metoclopramide with morphine and pethidine. $\mathrm{Br}$ J Anaesth 1974;46:514-9.

2 Assaf RAE, Dundee JW, Samuel IO. The efficacy of metoclopramide against narcotic-induced emetic symptoms. $\mathrm{Br} \mathcal{F} \mathrm{Clin}$ Pharmacol 1974;1:177p.

3 Dundee JW, Chestnutt WN, Ghaly RG, Lynas AGA. Reduction in emetic effects of opioid preanaesthetic medication by in emetic effects of opioid preanaesthetic medica

4 Dundee JW, Chestnutt WN, Ghaly RG, Lynas AGA. Traditional Chinese acupuncture: a potentially useful antiemetic? Br Med Chinese acupunctur

\section{Effect of breast conservation on} psychological morbidity associated with diagnosis and treatment of early breast cancer

SIR,-Dr Lesley J Fallowfield and others rightly state that there has been little systematic study of the psychosocial outcome of breast conservation (22 November, $p$ 1331). It is unfortunate that they could not carry out a prospective study. The retrospective design, however, presents a major problem, as measures of morbidity were taken only once over four to 32 months and the results pooled. Different stressors would be operating on the women interviewed four months postoperatively from those operating in women interviewed at 32 months. At four months the effects of radiotherapy cause depression but this is unlikely at 32 months. The study cannot answer the important question: Do women treated with lumpectomy and radiotherapy become depressed soon after operation but then improve?

The problem with much psychosocial research in oncology is that different measures are used and differing criteria applied for psychiatric "caseness." The authors use a well proved instrument, the present state examination, and then apply DSM III criteria to define psychiatric caseness. In previous papers Maguire has used the present state examination and applied other criteria. It is a pity that the CATEGO computer program designed to analyse symptoms exposed by the present state examination has not been used throughout his papers to facilitate comparability.

I have interviewed over 200 women in a similar, but prospective, conservation study carried out at the Breast Unit, Guy's Hospital, which is currently being analysed, and it is clear that their main worry relates to the implications of a diagnosis of cancer. Unfortunately this paper gives the impression, without presenting any quantifiable evidence, that women treated by lumpectomy worry more about recurrence. Similarly, the discussion suggests that the depression experienced by the women treated by lumpectomy relates to the radiotherapy. This conclusion cannot be made from the results that are presented because no attempt has been made to analyse the relation between morbidity and treatment variables.

I fear that some readers may have been left with the idea that breast conserving treatment produces the same or even greater psychiatric morbidity as mastectomy without consideration being given to 